\title{
Taxonomia dos objetivos educacionais e as teorias de aprendizagem no treinamento das técnicas cirúrgicas laparoscópicas em ambiente de simulação.
}

\section{Taxonomy of educational objectives and learning theories in the training of laparoscopic surgical techniques in a simulation environment.}

Grijalva Otávio Ferreira da Costa, TCBC-CE; Hermano Alexandre lima Rocha ${ }^{2}$; Luiz Gonzaga de Moura Júnior ${ }^{3}$; Francisco das Chagas Medeiros ${ }^{2}$

R E S U M O

\begin{abstract}
A aquisição de habilidades psicomotoras em cirurgia é o componente central dos programas de residência médica em Cirurgia Geral e Especialidades. Tornar o aprendizado mais efetivo é ponto basilar dos processos educacionais. Esse artigo retrata os aspectos das taxonomias educacionais e teorias de aprendizagem que podem ser envolvidas no treinamento da cirurgia. Entre as inúmeras taxonomias e teorias educacionais aplicáveis no aprendizado em cirurgia destacam-se: 1) Taxonomia de Dave- Hierarquização a ações que facilitam a aquisição de habilidades psicomotoras; 2) Teoria de MillerDefinição do passo a passo que facilita a aquisição das habilidades; 3) Teoria de Ericsson- Competência após a repetição da prática seguida de reforço sistemático; 4) Teoria de Vigotsky- Definição do papel do especialista no aprendizado; 5) Teoria de Boud, Schon e Ende- Importância da devolutiva (feedback) para alunos e professores. O conhecimento dessas ferramentas por professores e preceptores pode facilitar o aprendizado na cirurgia, em especial nas atividades mais complexas.
\end{abstract}

Descritores: Educação Médica. Treinamento por Simulação. Ensino. Laparoscopia.

\section{INTRODUÇÃO}

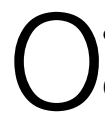
atual modelo de treinamento para a formação de cirurgiões no mundo ocidental foi proposto e implantado por William Halsted, no Hospital John Hopkins, em 1889, nos Estados Unidos. O modelo inicial introduziu o conceito de residência médica baseado na experiência germânica de treinamento para cirurgiões. Com o passar do tempo, esse modelo de treinamento foi adotado nos Estados Unidos e em toda a América, ${ }^{1,2}$.

Classicamente, essa formação é realizada na sala de operação sob a supervisão de um cirurgião graduado. Essa forma de ensino, embora eficiente, é demorada, tem alto custo e pode aumentar a morbidade para os pacientes ${ }^{3}$. Com o advento da cirurgia minimamente invasiva, nos anos 1980, os cirurgiões foram incentivados a adquirir uma série de novas habilidades para superar desafios técnicos até então inexistentes na prática cirúrgica convencional, como perda da percepção de profundidade e orientação espacial devido à visão em duas dimensões, percepção invertida dos movimentos das mãos no trabalho com instrumentos cirúrgicos (efeito fulcral da parede abdominal), limitado grau de movimentação em função do uso de instrumentos rígidos introduzidos por meios de trocartes fixos na parede abdominal, diminuição da sensibilidade háptica devido à resistência dentro dos trocartes e ao uso de instrumentos longos e a necessidade de realizar as habilidades com ambas as mãos (ambidestria) ${ }^{4-6}$.

O modelo de treinamento tradicional para o aprendizado em procedimentos cirúrgicos abertos mostrou-se, ao longo do tempo, ser inadequado e de baixa eficiência quando aplicado ao treinamento das habilidades em procedimentos laparoscópicos. Além disso, as habilidades cirúrgicas já adquiridas por cirurgiões em procedimentos cirúrgicos abertos na realização de nós cirúrgicos não facilitavam o aprendizado dos nós intracorpóreos laparoscópicos, sendo necessário para esses um

1 - Centro Universitário Christus, Curso de Medicina, Fortaleza, CE, Brasil. 2 - Universidade Federal do Ceará, Curso de Medicina, Fortaleza, CE, Brasil. 3 - Universidade Estadual do Ceará, Curso de Medicina, Fortaleza, Ceará, Brasil. 
treinamento específico em procedimentos cirúrgicos minimamente invasivo ${ }^{7}$. Com o passar dos anos ficou claro que o modelo de treinamento proposto por Halsted (see one, do one, teach one) não se aplicaria ao aprendizado das habilidades da cirurgia minimamente invasiva e teria que ser substituído por um modelo que priorizasse a simulação com muitas repetições e sempre sob supervisão qualificadå.

O aprendizado das técnicas cirúrgicas inclui obrigatoriamente a aquisição de diversas habilidades psicomotoras, definidas como atividades mentais e motoras requeridas para executar uma determinada tarefa manual ${ }^{9}$. Para facilitar o treinamento das novas habilidades psicomotoras, foram desenvolvidos simuladores e ambientes de simulação, eliminando, assim, os riscos de iatrogenias e ofertando aos aprendizes um local seguro, confortável e sem a pressão da sala de operação $0^{6,10}$.

Com a natural evolução da videocirurgia, os procedimentos abdominais tornaram-se cada vez mais complexos e aos poucos foram necessários a realização de nós, pontos e suturas intracorpóreas. Nas experiências iniciais, ficou evidente que essas manobras eram difíceis de ser realizadas, passando a ser consideradas manobras de alta complexidade ${ }^{9,11}$. Para suprir essa dificuldade, alguns modelos de treinamento para a realização de nós, pontos e suturas intracorpóreas em operações laparoscópicas foram propostos e aplicados ${ }^{10,12}$.

Um programa de treinamento em laparoscopia básica e avançada para residentes, preceptores e diretrizes para treinamento para aqueles que já haviam concluído a residência em Cirurgia, foi proposto, em 1998, pela Sociedade Americana de Cirurgia Gastrointestinal e Endoscopia (SAGES). O objetivo da proposta era incorporar o treinamento em laparoscopia avançada aos programas de treinamento das residências médicas em cirurgia ${ }^{13}$.

A Sociedade Americana de Cirurgia Gastrointestinal e Endoscopia, endossada pelo Colégio Americano de Cirurgiões (ACS), propôs, em
2004, um programa educacional de treinamento denominado Fundamental of Laparoscopic Surgery (FLS) com o objetivo de ensinar e avaliar as habilidades psicomotoras básicas requeridas na performance da cirurgia laparoscópica para residentes e cirurgiões. O programa baseia-se em uma avaliação cognitiva inicial e um treinamento prático realizado em simulador com cinco tarefas: transferir objetos, cortar tecido, aplicar ligadura com alça, realizar suturas extracorpórea e intracorpórea. O treinamento é fundamentado na repetição das tarefas propostas. O tempo de cada exercício é definido individualmente, com limite previamente estabelecido. Ao final do treinamento, é conferido certificação para os aprovados ${ }^{14}$.

Na Europa, a Sociedade Europeia de Cirurgia Endoscópica implementou um programa de treinamento para cirurgiões e residentes denominado Laparoscopic Surgical Skills (LSS). A estrutura desse programa é composta de três fases: pré-curso, curso e pós-curso, e todos incluem atividades teóricas e práticas ${ }^{15}$.

Em função da dificuldade de garantir um aprendizado efetivo aos cirurgiões, Sadideen et al. ${ }^{9}$ realizaram uma revisão dos aspectos das teorias educacionais que poderiam ser aplicáveis ao ensino da cirurgia. Entre elas foram consideradas as mais importantes: 1) aquisição e retenção de habilidades motoras (pirâmide de Miller; teoria de Fitts e Posner); 2) desenvolvimento de expertise com a prática repetida e reforço (teoria de Ericsson); 3) disponibilidade de supervisão com especialista (teoria de Vygotsky); 4) aprendizagem no ambiente de prática (teorias de Lave e Wenger); 5) feedback na aprendizagem de habilidades práticas (teorias de Boud, Schon e Ende); e 6) afetividade na aprendizagem ${ }^{9}$.

Assim como na capacitação de cirurgiões, a dos médicos residentes, nas técnicas de videocirurgia, é baseada, na maioria dos programas, em modelos de treinamento cientificamente não testados e sem a garantia da obtenção da competência no processo do saber fazer ao final do processo $^{16}$. No Brasil, 
esforços significativos têm sido feitos para melhorar o aprendizado das habilidades psicomotoras em procedimentos cirúrgicos com o desenvolvimento de modelos de treinamento inovadores, como os propostos por Artifon et al., modelo ex-vivo para o ensino avançado na colangiopancreatografia endoscópica retrógrada17, Oti et al., modelo de treinamento de cirurgia laparoscópica utilizando smartphone $^{18}$, spencer et al., modelo porcino de baixo custo para dissecção venosa ${ }^{19}$ e Cunha et al., que desenvolveram um simulador de baixo custo para treinamento em videocirurgia em três dimensões ${ }^{20}$. Moura Júnior et al. testaram um modelo de avaliação de desempenho em endossuturas em laboratório de simulação e concluíram que o modelo era seguro e revelava o perfil do aluno, assim como, a sua performance ao final do treinamento ${ }^{21}$.

Nacúl et al. realizaram uma análise crítica do atual do treinamento de médicos residentes em videocirurgia no Brasil e sugeriram que a capacitação para a aquisição de habilidades em videocirurgia nas residências médicas necessita de um processo pedagógico mais adequado de ensino para conferir uma base educacional mais sólida do que a atual ${ }^{22}$. Rasslan et al. avaliaram o perfil do residente de Cirurgia Geral do Hospital das Clínicas da Faculdade de Medicina de São Paulo e concluíram que há uma redução pela procura e uma definição mais precoce da especialidade. Considerando a duração de dois anos da residência médica em Cirurgia Geral é provável que o treinamento em videocirurgia seja restrito ${ }^{23}$.

A realização dessa revisão foi motivada pela observação da enorme dificuldade encontrada por médicos residentes, durante o aprendizado das habilidades laparoscópicas e principalmente na realização de nós, pontos e suturas. São objetivos centrais desse artigo a necessidade de compreender em que aspectos estão centradas a taxonomia dos objetivos educacionais e as teorias educacionais de aprendizagem aplicáveis aos modelos de treinamento em procedimentos cirúrgicos.

\section{TAXONOMIA DOS OBJETIVOS}

\section{EDUCACIONAIS}

Ensinar habilidades práticas é o componente central da educação cirúrgica de graduação e pósgraduação. De uma maneira geral, os modelos de aprendizagem das técnicas operatórias não são previamente testados e não há como assegurar a eficiência ao final do treinamento. A efetiva aprendizagem das habilidades psicomotoras requer uma abordagem que inclui vários componentes, entre os quais o meio ambiente (cenário de prática), a estruturação da prática, o diálogo professor/aluno e as estratégias pedagógicas ${ }^{24}$.

Nesse contexto, o conceito de aprendizado é definido como tornar-se apto ou capaz, mediante estudo, observação ou experiência, após tomar conhecimento, compreensão e retenção na memória. A habilidade é uma resposta comportamental na prática estabelecida pela repetição frequente e continuada de movimentos que ampliam a agilidade, a destreza, a precisão no manejo e na percepção sensitivo-motora. Já a aptidão é o ponto máximo da percepção sensitivo-motora obtida após o treinamento, enquanto a proficiência é quando a aptidão, competência e retenção de habilidade atingem uma estabilidade, um platô ${ }^{25}$. Embora amplamente citado em diversas pesquisas, os critérios de proficiência em realizar pontos e suturas laparoscópicas não foi definida por nenhuma ${ }^{26,27}$.

Para facilitar o planejamento, organização e o controle dos objetivos de aprendizagem, Benjamim Bloom propôs uma classificação estruturada e orientada ${ }^{28}$. Essa classificação, conhecida como Taxonomia de Bloom, serviu como base para pesquisadores que a adaptaram e a aperfeiçoaram. A taxonomia de Bloom oferece vantagens como: estabelecer uma linguagem comum acerca dos objetivos de aprendizado, servir de base para o desenvolvimento de instrumentos de avaliação, estimular o desempenho dos alunos, 
incentivar educadores a auxiliarem aos seus alunos de forma estruturada e consciente a adquirirem competências específicas e determinar coerências entre os objetivos educacionais, as atividades e as avaliações nos currículos 29,30 .

Dave, em 1967, propôs uma taxonomia de objetivos educacionais para facilitar a aquisição das habilidades psicomotoras e a escalonou em: imitação, manipulação, precisão prática, articulação e naturalização ${ }^{24}$. Essa classificação foi amplamente utilizada como diretriz educacional pelo programa ATLS (Advanced Trauma Life Suport), do American College of Surgeons, nos últimos 30 anos. Simpson definiu que o domínio psicomotor consiste no movimento somático, na coordenação motora e no uso das áreas psicomotoras. O desenvolvimento dessas habilidades requer treinamento prático que, em geral, é medido em velocidade, precisão ou desempenho técnico na execução. Os princípios do aprendizado das habilidades psicomotoras foram definidos em conceptualização, visualização, verbalização, prática, correção e reforço, domínio da habilidade e autonomia. Esses princípios devem ser claros e dispostos de maneira sequencial e hierárquica nos modelos de treinamento para a aquisição de habilidades psicomotoras ${ }^{24}$.

\section{TEORIAS EDUCACIONAIS DE}

\section{APRENDIZAGEM}

A compreensão das teorias educacionais de aprendizagem por parte de preceptores de programas de cirurgia e professores da graduação pode facilitar a estruturação e aplicação de modelos de treinamento em procedimentos e técnicas operatórias. Inúmeras são as teorias de aprendizagem para a aquisição de habilidade aplicáveis à aquisição de habilidades psicomotoras em procedimentos cirúrgicos, entre as quais, destacam-se: teoria de Miller, teoria de Fitts e Posner, Ericsson, Vygotsky, Lave e Wenger, Boud, Schon e Ende.
Miller propôs uma sequência hierárquica de competência em quatro níveis, tendo como base o "saber", seguido do "saber como", "demonstrar como" e, finalmente, o "fazer". Dessa forma, estabeleceu o passo a passo para a competência, no qual o aprendiz avança por meio dos passos cognitivos e comportamentais necessários que estão subjacentes ao próximo passo, construindo o conhecimento que eventualmente auxilia e suporta a execução de uma habilidade específica. Há, aparentemente, uma desvantagem nesse processo que assume que a competência incluiu previamente o conhecimento ${ }^{31}$.

Fitts e Posner estabeleceram uma teoria para a aquisição de habilidades motoras em três fases: cognitiva (quando a habilidade é aprendida), associativa (quando o desempenho se aproxima da habilidade) e a da autonomia (quando a habilidade se tornou totalmente automática e pode ser realizada sem pensar muito sobre a tarefa). No estágio cognitivo, o aprendiz intelectualiza a tarefa. Com a prática contínua e o feedback relevante, atinge-se o estágio associativo ou integrativo, durante o qual o conhecimento é traduzido em comportamento motor apropriado. E, finalmente, a prática contínua resulta em um desempenho mais qualificado na fase de autonomia, no qual o estagiário não pensa em como está executando e começa a se concentrar nos demais aspectos relacionados à habilidade proposta ${ }^{32}$.

Vygotsky, psicólogo russo do início do Século $X X$, definiu com precisão o papel dos especialistas na assistência. Ele sugeriu a noção de uma "zona de desenvolvimento proximal", dentro da qual o aluno poderia progredir na resolução de problemas "em colaboração com pares mais capazes", mesmo sendo ele incapaz de fazê-lo de forma independente ${ }^{33}$. Cada aluno tem a sua "zona de desenvolvimento proximal". Alguns aprendizes começam em um nível mais avançado, enquanto outros não. A "zona de desenvolvimento proximal " de cada estudante pode variar, exigindo diferentes níveis de suporte a pares e instruções do orientador, até que eventualmente a habilidade possa ser dominada ${ }^{34}$. 
Lave e Wenger definiram que o aprendizado é um aspecto inseparável e integrado à prática social, em vez de um processo de internalização de uma experiência individual. Assim, o componente essencial do aprendizado, quando visto como uma atividade, é o processo de participação. Isso significa que os alunos que integram comunidades de práticas, com o objetivo de dominar habilidades, devem avançar para a plena participação nas práticas socioculturais dessa comunidade. Esse processo social pode incluir a aprendizagem de habilidades práticas. Fica claro que a participação do aprendiz é crucial nesta teoria, uma vez que há o seu envolvimento com pares no estágio comum. Essa teoria não está diretamente relacionada aos cuidados de saúde, entretanto, pode se notar que, para adquirir habilidades com sucesso, é necessária uma interação social sustentada, que é usualmente demorada. Obviamente, o melhor é começar a aprender as habilidades práticas simples e de baixo risco, durante as quais os aprendizes podem atingir metas plausíveis ${ }^{35}$.

Boud e Schon descreveram processos pelos quais os formandos aprendem com a prática o saber, a aprendizagem experiencial e a reflexão sobre a prática (feedback). O feedback pode ser aplicado após a realização da sessão de ensino, ao executar a habilidade ou antes da ação. A combinação de todos os processos de feedback pode maximizar o processo de reflexão ${ }^{36,37}$. Para Ende, o feedback dos formadores (professores, preceptores) é tão importante quanto o feedback dos próprios aprendizes $^{38}$. O feedback é considerado uma das mais potentes ferramentas do aprendizado, sendo útil no desenvolvimento e no direcionamento das etapas subsequentes. Assim, o feedback é um componente crucial do aprendizado de habilidades práticas, seja aquele definido pela abordagem Vygotskiana, na teoria de Lave e Wenger, ou na prática deliberada, ajudando o aluno a obter conhecimentos especializados.

O componente afetivo na aprendizagem não pode ser esquecido nesse processo. Ele é poderoso e exerce efeitos tanto positivos como negativos nas experiências dos aprendizes e, em alguns aspectos, é fundamental para a aquisição de habilidades psicomotoras. Não é incomum que pessoas mais velhas reportem experiências que foram enriquecedoras ou desastrosas e que afetaram significativamente o seu desenvolvimento profissional ${ }^{39}$. O modelo hierárquico no qual os aspectos físicos, emocionais e psicológicos dos aprendizes precisam ser solucionados antes do início do aprendizado foi descrito por Maslow, estabelecendo como condição essencial para o aprendizado a criação de um ambiente sustentável e agradável, com o objetivo de motivar e incentivar a participação no processo de aprendizagem ${ }^{40}$.

\section{CONCLUSÃO}

Diante dos desafios de promover um aprendizado efetivo para a aquisição de habilidades psicomotoras parece sensato embasar os modelos de treinamento das técnicas operatórias, e em especial o da realização de nós e pontos laparoscópicos, em taxonomia de objetivos educacionais e teorias de aprendizagem.

\section{A B S T R A C T}

The acquisition of psychomotor skills in surgery is the central component of medical residency programs in General Surgery and Specialties. Making learning more effective is a cornerstone of educational processes. This article portrays aspects of educational taxonomies and learning theories that may be involved in the training of surgery. Among the many taxonomies and educational theories applicable to learning in surgery, the following stand out: 1) Dave's taxonomy- Hierarchy to actions that facilitate the acquisition of psychomotor skills; 2) Miller's theoryStep-by-step definition that facilitates acquisition; 3) Ericsson's theory-Competence after repetition of the practice followed by systematic reinforcement; 4) Vigotsky's theory- Definition of the role of the specialist in learning; and 5) Theory of Boud, Schon and Ende- Importance of feedback for students and teachers. Knowledge of these tools by teachers and preceptors can facilitate learning in surgery, especially in more complex activities.

Keywords: Education. Medical. Simulation Training. Teaching. Laparoscopy. 


\section{REFERÊNCIAS}

1. Cameron JL. William Stewart Halsted. Our surgical heritage. Ann Surg. 1997;225(5):445-8.

2. Kerr B, O'Leary JP. The training of the surgeon: Dr. Halsted's greatest legacy. Am Surg. 1999;65(11):1101-2.

3. Bridges $M$, Diamond DL. The financial impact of teaching surgical residents in the operating room. Am J Surg. 1999;177(1):28-32.

4. Gallagher AG, McClure N, McGuigan J, Ritchie K, Sheehy NP. An ergonomic analysis of the fulcrum effect in the acquisition of endoscopic skills. Endoscopy. 1998;30(7):617-20.

5. Anastakis DJ, Regehr G, Reznick RK, Cusimano M, Murnaghan J, Brown M, et al. Assessment of technical skills transfer from the bench training model to the human model. Am J Surg. 1999;177(2):167-70.

6. Scott DJ, Bergen PC, Rege RV, Laycock R, Tesfay ST, Valentine RJ, et al. Laparoscopic training on bench models: better and more cost effective than operating room experience? J Am Coll Surg. 2000;191(3):272-83.

7. Figert $P L$, Park $A E$, Witzke $D B$, Schwartz RW. Transfer of training in acquiring laparoscopic skills. J Am Coll Surg. 2001;193(5):533-7.

8. Satava RM. Emerging trends that herald the future of surgical simulation. Surg Clin North Am. 2010;90(3):623-33.

9. Sadideen $\mathrm{H}$, Kneebone R. Practical skills teaching in contemporary surgical education: how can educational theory be applied to promote effective learning? Am J Surg. 2012;204(3):396-401.

10. Derossis AM, Fried GM, Abrahamowicz M, Sigman $\mathrm{HH}$, Barkun JS, Meakins JL. Development of a model for training and evaluation of laparoscopic skills. Am J Surg. 1998;175(6):482-7.

11. den Boer KT, de Jong T, Dankelman J, Gouma DJ. Problems with laparoscopic instruments: opinions of experts. J Laparoendosc Adv Surg Tech A. 2001;11(3):149-55.

12. Rosser JC, Rosser LE, Savalgi RS. Skill acquisition and assessment for laparoscopic surgery. Arch Surg. 1997;132(2):200-4.
13. Integrating advanced laparoscopy into surgical residency training. Surg Endosc. 1998;12(4):374-6.

14. Okrainec A, Soper NJ, Swanstrom LL, Fried GM. Trends and results of the first 5 years of Fundamentals of Laparoscopic Surgery (FLS) certification testing. Surg Endosc. 2011;25(4):1192-8.

15. Buzink S, Soltes $M$, Radonak J, Fingerhut $A$, Hanna G, Jakimowicz J. Laparoscopic Surgical Skills programme: preliminary evaluation of Grade I Level 1 courses by trainees. Wideochir Inne Tech Maloinwazyjne. 2012;7(3):188-92.

16. Roberts KE, Bell RL, Duffy AJ. Evolution of surgical skills training. World J Gastroenterology. 2006;12(20):3219-24.

17. Artifon EL, Nakadomari TS, Kashiwagui LY, Belmonte EA, Solak CR, Cheng $S$, et al. An innovative ex-vivo model for rapid change of the papilla for teaching advanced endoscopic retrograde cholangiopancreatography procedures. Arq Bras Cir Dig. 2016;29(4):269-71. English, Portuguese.

18. Oti AT, Galvão LN, Pessoa TCP, Rocha CRO, Monteiro AM, Fonteles MJP, et al. Development of a laparoscopic training model using a smartphone. Rev Col Bras Cir. 2017;44(5):471-5. English, Portuguese.

19. Spencer FAC Netto, Silva MTB, Constantino MM, Cipriani RFF, Cardoso M. Educational project: low cost porcine model for venous cutdown training. Rev Col Bras Cir. 2017;44(5):545-8. English, Portuguese.

20. Cunha CMQD, Lima DMF, Menezes FJC. Low-cost simulator assembly for 3-dimensional videosurgery training. Arq Bras Cir Dig. 2018;31(3):e1384. English, Portuguese.

21. Moura-Júnior LG, Ramos A, Campos JM, Ferraz ÁA, Rocha HÂL, Costa GO. Teaching model for evaluation of the ability and competence progress in endosuture in surgical skill laboratory. Arq Bras Cir Dig. 2017;30(4):256-9. English, Portuguese.

22. Nácul MP, Cavazzola LT, Melo MC. Situação atual do treinamento de médicos residentes em videocirurgia no Brasil: uma análise crítica. Arq Bras Cir Dig. 2015;28(1):81-5.

23. Rasslan S, Arakaki MS, Rasslan R, Utiyama EM. Profile of the General Surgery resident: what are the changes in the 21st Century? Rev Col Bras Cir. 2018;45(2):e1706. English, Portuguese. 
24. Simpson E. The classification of educational objectives in the psychomotor domain: The psychomotor domain. Washington, DC: Gryphon House; 1972.

25. Ferreira $A B H$. Novo dicionário da língua portuguesa. Rio de Janeiro: Nova Fronteira; 2012.

26. Dehabadi $M$, Fernando $B$, Berlingieri P. The use of simulation in the acquisition of laparoscopic suturing skills. Int J Surg. 2014;12(4):258-68.

27. Nickel F, Hendrie JD, Kowalewski KF, Bruckner T, Garrow CR, Mantel $M$, et al. Sequential learning of psychomotor and visuospatial skills for laparoscopic suturing and knot tying-a randomized controlled trial "The Shoebox Study" DRKS00008668. Langenbecks Arch Surg. 2016;401(6):893-901.

28. Bloom BS, editor. Taxonomy of educational objectives: the cognitive domain. New York: Logman; 1956.

29. Ferraz APCM, Belhot RV. Taxonomia de Bloom: revisão teórica e apresentação das adequações do instrumento para definição de objetivos instrucionais. Gest Prod. 2010;17(2):421-31.

30. Krathwohl DR. A revision of Bloom's taxonomy: an overview. Theory Pract. 2002;41(4):212-8.

31. Miller GE. The assessment of clinical skills/competence/ performance. Acad Med. 1990;65(9 Suppl):S63-7.

32. Reznick RK, MacRae $H$. Teaching surgical skills-changes in the wind. N Engl J Med. 2006;355(25):2664-9.

33. Vygotsky L. Thought and language. Cambridge (MA): MIT Press; 1962.
34. Wertsch JV. Vygotsky and the social formation of mind. Cambridge (MA): Harvard University Press; 1985.

35. Lave J, Wenger E. Situated learning: legitimate peripheral participation. Cambridge: Cambridge University Press; 1991.

36. Boud D. A facilitator's view of adult learning. In: Bould D, Griffin V, editors. Appreciating adults learning: from the learners' perspective. London: Kogan Page; 1987. p.222-39.

37. Schön DA. The reflective practitioner: how professionals think in action. Nova York: Basic Books; 1983.

38. Ende J. Feedback in clinical medical education. JAMA. 1983;250(6):777-81.

39. Cassar K. Development of an instrument to measure the surgical operating theatre learning environment as perceived by basic surgical trainees. Med Teach. 2004;26(3):260-4.

40. Monkhouse $S$. Learning in the surgical workplace: necessity not luxury. Clin Teach. 2010;7(3):167-70.

Recebido em: 13/06/2018

Aceito para publicação em: 09/09/2018

Conflito de interesse: nenhum.

Fonte de financiamento: nenhuma.

\section{Endereço para correspondência:}

Grijalva Otávio Ferreira da Costa

E-mail: grijalvafc@uol.com.br internato01@unichristus.edu.br 